\title{
Modes of energy transfer from the solar wind to the inner magnetosphere
}

\author{
D. Vassiliadis ${ }^{\text {a) }}$ \\ Universities Space Research Association, NASA/Goddard Space Flight Center, Greenbelt, Maryland 20771
}

R. S. Weigel

National Research Council at NASA/Goddard Space Flight Center, Greenbelt, Maryland 20771

A. J. Klimas

Laboratory of Extraterrestrial Physics, NASA/Goddard Space Flight Center, Greenbelt, Maryland 20771

S. G. Kanekal' ${ }^{\text {b) }}$

Laboratory for Astrophysics and Space Physics, University of Colorado, Colorado

\author{
R. A. Mewaldt \\ California Institute of Technology, Pasadena, California
}

(Received 19 June 2002; accepted 7 November 2002)

\begin{abstract}
Energy transport from the interplanetary plasma to Earth's inner magnetosphere occurs in a range of time scales and efficiencies. It is often hypothesized that this range is smoothly varying with radial geocentric distance, indicating the transport involves many processes, whose ranges overlap. Here we report evidence from observations, and time series analysis, and other data-based modeling which indicates that the coupling of magnetospheric relativistic electron fluxes to solar wind variables occurs in specific ranges of radial distance ( $L$ shell). These findings probably have important consequences for the understanding of physical mechanisms responsible for the acceleration in each region. We identify three distinct regions: $P_{0}$ at approximately $3<L<4 R_{E}, P_{1}$ at $4<L<7 R_{E}$, and $P_{2}$ at $L>7 R_{E}$. Each one responds to a different combination of solar wind variables, and couples to the main driver variable, the solar wind speed $V_{\mathrm{SW}}$, in a different way. Mode $P_{1}$ is the prototypical response of the inner magnetosphere. The electron flux responds more slowly than the other two regions to $V_{\mathrm{SW}}(2-3$ days): high-speed streams are the most geoeffective structures for that region. Mode $P_{0}$ responds significantly faster ( $<1$ day) and seems to be more affected by the negative $B_{z}$ component of the interplanetary field (probably through magnetic reconnection) and the magnitude of the field, rather than by variations in solar wind plasma variables. Region $P_{2}$ contains much lower fluxes of trapped particles than the other two, and responds rapidly ( $\sim 1$ day) to positive $B_{z}$ and to lower solar wind speed. The interpretation is that these regions are representative of different modes of energy transfer from the interplanetary medium to the inner magnetosphere with implications for very different particle acceleration mechanisms. (C) 2003 American Institute of Physics. [DOI: 10.1063/1.1535938]
\end{abstract}

\section{INTRODUCTION}

Modern space plasma physics has shifted its focus from studying plasmas in isolation to understanding the interactions between two or more adjacent plasmas. This development is motivated by the large variety of interacting plasma systems in the geospace environment (interplanetary space, magnetosphere, ionosphere, and their subdivisions) and facilitated by the explosive increase in high-quality, multispectral observational data. This paper examines the interaction between two very different plasmas, the interplanetary medium and the inner magnetosphere. The solar wind is a turbulent, radially streaming magnetofluid with $\beta \leqslant 1$ while the radiation belts are characterized primarily by particle and kinetic effects where the ambient field geometry and plasma

\footnotetext{
${ }^{a)}$ Electronic mail: vassi@electra.gsfc.nasa.gov

${ }^{b}$ Present address: Department of Physics, Catholic University of America.
}

distribution are important. Transport of energy and momentum (and to a lesser extent of density) takes place continually between these two media.

The present work will suggest that a significant part of this momentum/energy transfer to the inner magnetosphere is organized in specific modes. They are the net products of many simultaneously operating physical mechanisms some of which may dominate at particular spatial locations in the inner magnetosphere. We will discuss time series analysis and modeling methods which are useful in determining the temporal and spatial scales of the modes. The long-term response, as well as solar-cycle and seasonal dependencies have been examined elsewhere. ${ }^{1}$ These features can then be used to identify the modes with individual physical processes or groups thereof.

The physics of the modes of response is related to types of particle acceleration and the formation of the radiation belts. ${ }^{2}$ Modeling processes of acceleration as well as trapping is important in understanding some of the most explosive events in the magnetospheric plasma environment. Part of 


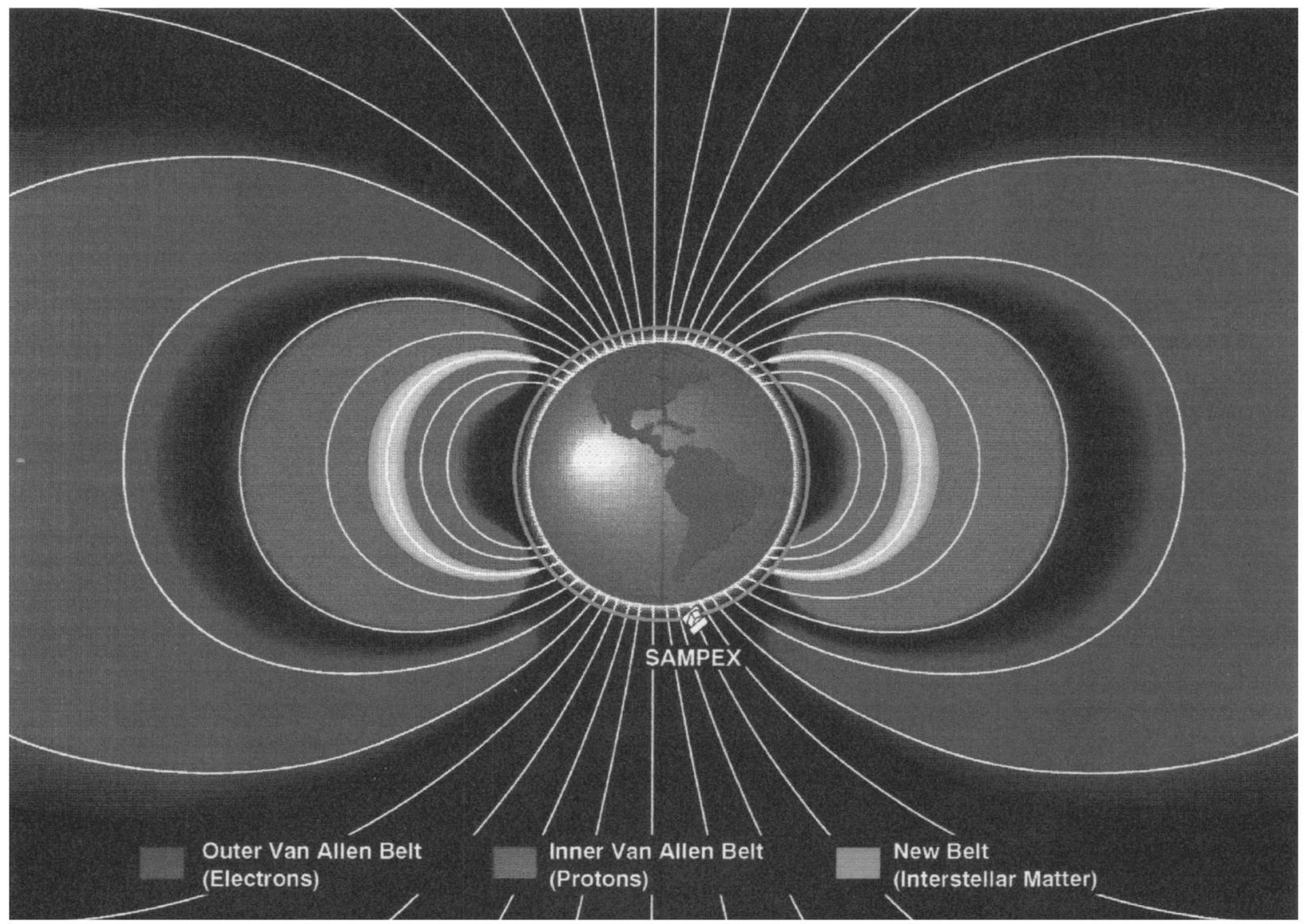

FIG. 1. Schematic view of the electron radiation belts. The inner and outer zones are quasitoroidal regions whose meridian cross sections are seen here. The radial structure of the outer zone and energy transport to it from the solar wind is the subject of this paper. A third, transient belt with an unexpectedly high lifetime of 6 months was identified by the SAMPEX spacecraft in 1994. The orbit of SAMPEX is shown schematically.

the challenge is the fact that the energization processes occur wholly within the magnetosphere, i.e., the majority of highenergy particles do not originate in the solar wind. ${ }^{3}$ Electrons, in particular, reach relativistic energies and are then trapped to form the radiation belts.

Trapping and its stability, on the other hand, are determined by the large-scale properties of the magnetic field. This paper will suggest that each radial region in the radiation belts is characterized by different particle acceleration mechanisms in sofar as this is determined from the coupling to the solar wind. Here long-term trapping is effectively used as a probe of the spatial structure of the innermagnetospheric field. Because energetic particles are characterized by rapid gyration and bounce motion along field lines, two of their coordinates can be neglected for timescales longer than a few hours, and their position is often quoted in terms of the geocentric radial distance. This parameter, denoted by $L$, is the geocentric distance on the equatorial plane of the footpoint of the field line passing through the particle location,

$$
R=L \cos ^{2} \lambda,
$$

where $R$ and $\lambda$ are the polar coordinates (geocentric distance and latitude) of the particle position on the meridian plane of the field line; $L$ and $R$ are measured in Earth radii, $R_{E}$; and the model field is often dipole so the field is toroidal-like (Fig. 1), but higher-order fields are also used. Conventionally the radiation belts extend in the approximate range $L$ $=1-10 R_{E}$, although in the dayside that extent is limited by the magnetopause boundary. The dipole model becomes insufficient for distances $L \gg 10 R_{E}$ and/or disturbed conditions, for both of which the $L$ parameter is less meaningful. We will show how this range consists of different subregions that respond differently to the external forcing of the solar wind.

The range of energies attained during acceleration is impressive for a planetary plasma environment. Thermal magnetospheric electrons have energies from 10 to $100 \mathrm{eV}$ (quiet plasma sheet) to several or tens of $\mathrm{keV}$ (hot plasma sheet). These low-energy particles are accelerated to $\mathrm{MeV}$ energies in the inner magnetosphere during several-day-long storm periods, in which the fluxes of $\mathrm{MeV}$ electrons rise from the baseline values of $10^{2} \cdot$ particles $/ \mathrm{cm}^{2} / \mathrm{sr} / \mathrm{s}$ to $>10^{4}$ - particles $/ \mathrm{cm}^{2} / \mathrm{sr} / \mathrm{s}$ within 2-3 days or less, depending on mechanisms and location. Acceleration as well as energy loss take place continually in the radiation belts.

Several acceleration mechanisms have been put forth to account for these rapid flux increases. ${ }^{2,4}$ While the majority of them is probably physically plausible, their relative sig- 
nificance is not well known at this point in time. Some of the most important acceleration mechanisms fall into three relatively broad categories:

(1) Resonant particle acceleration: Wave-particle interactions are ubiquitous in the radiation belts. Ultra-lowfrequency (ULF) waves in the Pc5 range (2.5-10 min) precede electron growth by approximately 1 day. ${ }^{5}$ Global MHD simulations support the notion that the lowest modes of the wave electric field can accelerate particles. ${ }^{6}$ Recently the simulations have become increasingly realistic in terms of global dynamics and the magnetic field symmetry. $^{7}$

In terms of the coupling to the solar wind, increases in solar wind speed seem to contribute significantly in the wave excitation. ${ }^{8,9}$ Early on it was recognized that among the solar wind parameters, the bulk speed $V_{\mathrm{SW}}$ was the most closely related to the observed enhancement of particle fluxes. ${ }^{9}$ For example, high-speed streams in the solar wind are generally followed by strong electron storms. The speed enhancements induce wave activity in the magnetospheric flanks, probably through a viscous-type interaction. ${ }^{10}$ We will discuss this dependence on $V_{\mathrm{SW}}$ below, in terms of mode $P_{1}$.

(2) Rapid injection deep in the magnetosphere: Magnetic clouds are high-field, low-density transient structures in the solar wind. ${ }^{11}$ During the passage of magnetic clouds in January and May 1997, rapid accelerations took place much deeper in the magnetosphere, at $L \leqslant 4 R_{E},{ }^{12,13}$ than in the regions consistent with the ULF-wave induced acceleration. Electrons were energized in tens of minutes or in hours rather than in days. The physical mechanism leading to this condition is not as well understood as that of the previous case, but since the phenomenon was originally recognized, several events have been identified. This type of acceleration is probably very closely related to mode $P_{0}$ identified below.

(3) Shock-drift acceleration: This is a rare type of acceleration, having been clearly identified only once to date. The beginning of a magnetic storm (which is related to the ring current and is not necessarily accompanied by an electron storm) is often marked by the advent of a shock in the solar wind. This condition is called "storm sudden commencement" (SSC). During an unusually powerful SSC in March 1991 a radiation belt was formed within minutes at a relatively small distance, $L$ $=2.5 R_{E}$. In this case the new belt's lifetime exceeded 6 months. Its creation is adequately explained by the compression of the magnetosphere and the drift acceleration by the enhanced internal electric field. ${ }^{14}$ This rare type of acceleration has not been identified in our analysis.

In addition, several other mechanisms have been proposed (see, e.g., Ref. 4). For any given electron storm, many or all of these mechanisms probably operate simultaneously. It is not straightforward to separate a given storm into its constituent mechanisms. Instead we use a statistical approach: a large number of storms is modeled and common characteristics are identified. Then we make the correspondence between these characteristics and effects due to physical mechanisms.

\section{STRONGLY INTERACTING PLASMAS: MODES OF ENERGY TRANSPORT}

As is clear from the preceding discussion, the interaction between the solar wind plasma and the inner magnetosphere is multifaceted and many of the constituent mechanisms are nonlinear. Perhaps the most salient feature of the nonlinear response is that a linear increase in solar wind speed gives rise to exponentially higher fluxes (e.g., Ref. 9). In reality, as solar wind speed is elevated, either in magnitude or in fluctuation level, different types of dynamics are turned on and off. The effects of other parameters such as the $B_{z}$ component of the interplanetary magnetic field (IMF) and the plasma density $\rho$ are also of significance, especially for certain $L$ shell regions as will be seen below. Seasonal and solar-cycle effects are also important to include.

In spite of these complexities, a linear approximation to the coupling is useful because it helps identify some of the major interaction features and provides a reference point for more complex analyses. Therefore we will represent the interaction by a simple linear coupling between the solar wind speed $V_{\mathrm{SW}}$ (input or driver) and the relativistic electron flux at shell $L, j_{L}(t)=j(t ; L)$ (output). The quantity $j(t ; L)$ is the daily logarithmic omnidirectional flux of electrons in the range $2-6 \mathrm{MeV}$ at shell $L$. As mentioned, the quite-time flux is of the order of $10^{2}$ particles $/ \mathrm{cm}^{2} / \mathrm{sr} / \mathrm{s}$. The electron flux is measured by the Proton/Electron Telescope (PET) of the Solar, Anomalous, and Magnetospheric Particle Explorer (SAMPEX). ${ }^{15,16}$ Some of the basic properties of the spacecraft orbit, the instrument, and measurement data are given by Vassiliadis et al. ${ }^{1}$

The solar wind speed $V_{\mathrm{SW}}$ is also examined at a daily resolution. Its long-term average value is $\left\langle V_{\mathrm{SW}}\right\rangle=435 \mathrm{~km} / \mathrm{s}$ for the period 1993-2000 which constitutes the length of our database. High-speed streams, the most globally geoeffective transient structures (globally geoeffective refers to the entire inner magnetosphere), can be as fast as $800 \mathrm{~km} / \mathrm{s}$. For reference, the average properties of the solar wind plasma and interplanetary magnetic field (IMF) are summarized below:

Average Solar Wind Conditions,

$$
\begin{gathered}
\left\langle\rho_{\mathrm{SW}}\right\rangle=8.80 \mathrm{No} / \mathrm{cm}^{3} \\
\left\langle V_{\mathrm{SW}}\right\rangle=435 \mathrm{~km} / \mathrm{s}, \\
\left\langle P_{\mathrm{SW}}\right\rangle=2.573 \mathrm{nPa}, \\
\left\langle B_{z}\right\rangle=0, \\
\langle|B|\rangle=4.03 \mathrm{nT} .
\end{gathered}
$$

Measurements of the solar wind speed and other interplanetary variables are obtained from the OMNI database at NASA's NSSDC center.

At the linear approximation, the driving of $j(t ; L)$ by $V_{\mathrm{SW}}(t)$ is modeled as

$$
j(t ; L)=C+\int_{-T_{s}}^{T} H(\tau ; L) V_{\mathrm{SW}}(t-\tau) d \tau+\varepsilon(t),
$$


LONG-TERM-AVERAGE IMPULSE RESPONSE OF j(t; L=const.) TO $\mathrm{V}_{\text {SW }}$ 1993-2000

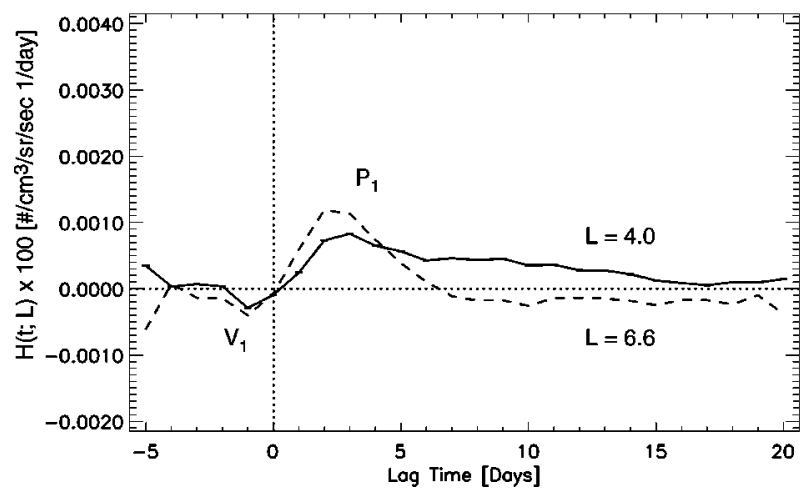

FIG. 2. Individual impulse response functions $H(t ; L)$ from Eq. (3) for energetic electron fluxes at $L=4.0$ and 6.6. The zero lag ( $\tau=0$ days) represents the time of impact of the solar wind. The response peaks $2-3$ days after that. Both of these orbits are part of region $P_{1}$ as discussed in the text.

where $C$ is a constant (typically taken equal to zero assuming the electron storm starts from a quiet state) and $\varepsilon(t)$ is the residual of the fit. The impulse response function $H(t ; L)$ relates the input from day $(t-T)$ to day $\left(t+T_{s}\right)$ to the output on day $t$. The function represents the various acceleration, transport, and loss processes that are important at shell $L$. Given a solar wind "gust" which can be approximated by a delta function, the expected output at shell $L$ is simply proportional to the impulse response function. The time $T_{s}$ is typically set to a small positive number $\left(T_{s}=5\right.$ days).

The linear model (3) can be obtained from a linear differential equation for the flux,

$$
\sum_{i=0}^{m-1} a_{i}^{(L)} \frac{d^{i} j_{L}}{d t^{i}}=V_{\mathrm{SW}}(t)
$$

where $m$ is typically a small number (e.g., $m=3$ ). In thatcase the impulse response function $H(t ; L)$ is a function of the $a_{i}^{(L)}$ in (4). Under certain conditions, where simple algebraic relations hold between the $a_{i}$ 's, the reverse is also true, namely one can obtain a linear model of the type (4) from $H(\tau ; L)$. Therefore, although we are not going to investigate the differential-equation model here, we will discuss findings for the impulse response and the most geoeffective solar wind properties.

The impulse response function for a given $L$ shell can be obtained by solving (3) using singular value decomposition for an overdetermined system, ${ }^{17}$ or a similar numerical method. The function is shown in Fig. 2 for $L=4.0$ and 6.6 $R_{E}$ (see also Ref. 1).

At both radial distances the solar wind impact at lag time $\tau=0$ is followed in approximately $2-3$ days by an increase in the response function. Following the peak response, denoted by $P_{1}$, the flux decays to zero, approximately exponentially. A second notable feature is the negative response at negative lag times, $\tau=-1$ day, denoted by $V_{1}$. It probably represents the adiabatic compression of the magnetosphere by an increasing solar wind pressure which leads to loss, radial transport, and other effects for the electrons at these $L$ shells. The response function for the geosynchronous region is consistent with earlier studies. ${ }^{18-20}$

Missions like SAMPEX have been invaluable in that they provide synoptic views of the entire radiation belt region for long intervals of time. Thus it is possible to synthesize the impulse responses for individual $L$ shells into a function parametrized by radial distance, as shown in Fig. 3 (see also Ref. 1). The two $L$ shells corresponding to Fig. 2 are denoted by dotted lines.

The two-dimensional response function has two peaks, denoted by $P_{0}$ and $P_{1}$. The latter peak is readily identified as the response at $\tau=2-3$ days after the solar wind impact extending over a wide radial range, $L=4-10 R_{E}$. This is the response to speed increases, well known from observations at geosynchronous orbit. ${ }^{9}$ The impulse response turns positive more or less simultaneously for a wide range of distances. The end time occurs much earlier for higher $L$ shells $\left(L>5 R_{E}\right)$ than for lower ones. In fact the end time is a piecewise linear function of the $L$ shell. ${ }^{1}$

The response at $P_{0}$, on the other hand, occurs at smaller radial distances, $L=3-4 R_{E}$. The response time is significantly faster, $0<\tau<1$. Its amplitude is, on average, much lower than the $P_{1}$ amplitude. We have found, however, that as the activity level of $j(t ; L)$ increases, the significance of $P_{0}$ grows much faster than, and eventually overtakes, that of $P_{1} \cdot{ }^{21}$

Nonlinear approximations to the $V_{\mathrm{SW}}$ driving can be made using artificial neural networks. These functions have already been applied to modeling and prediction of relativistic electron fluxes at the geosynchronous orbit. ${ }^{22}$ One of us (R.S.W.) has repeated the linear filter analysis of (3) with neural networks. Impulse response functions derived by linearizing the neural networks are strikingly similar to the impulse response functions corresponding to Fig. 3.

Interpretation: The modes obtained from the impulseresponse analysis are now related to the framework that is being developed for understanding particle acceleration in the radiation belts.

The $P_{1}$ mode is consistent with the response observed by many missions at geosynchronous distances. ${ }^{18-20,22}$ It is also consistent with the growth of ULF waves one day prior to the main energization of electrons as observed by SAMPEX/PET. ${ }^{5}$ ULF waves have been associated with the wave-induced acceleration of electrons to $\mathrm{MeV}$ energies. ${ }^{6,7}$ Also ULF waves are probably excited by solar wind speed increases ${ }^{8}$ such as during high speed streams that are known to induce particle acceleration. ${ }^{9}$ Thus the $P_{1}$ mode is most probably related to wave-induced particle acceleration. This does not exclude other mechanisms (such as recirculation or strong diffusion), but it excludes certain others (e.g., substorm-related injections probably occur at smaller time lags, $\tau<1$, and are not represented by this mode).

On the other hand, the $P_{0}$ mode is a rapid response which occurs at $L<4 R_{E}$. It occurs within the first day after solar wind impact. Thus it is consistent with the recent observations for rapid acceleration at those altitudes within a few hours from impact. ${ }^{12,13}$ We believe that this mode responds to a different combination of solar wind inputs than 
LONG-TERM-AVERAGE IMPULSE RESPONSE OF j(t;L) TO $V_{S W}$ 1993-2000

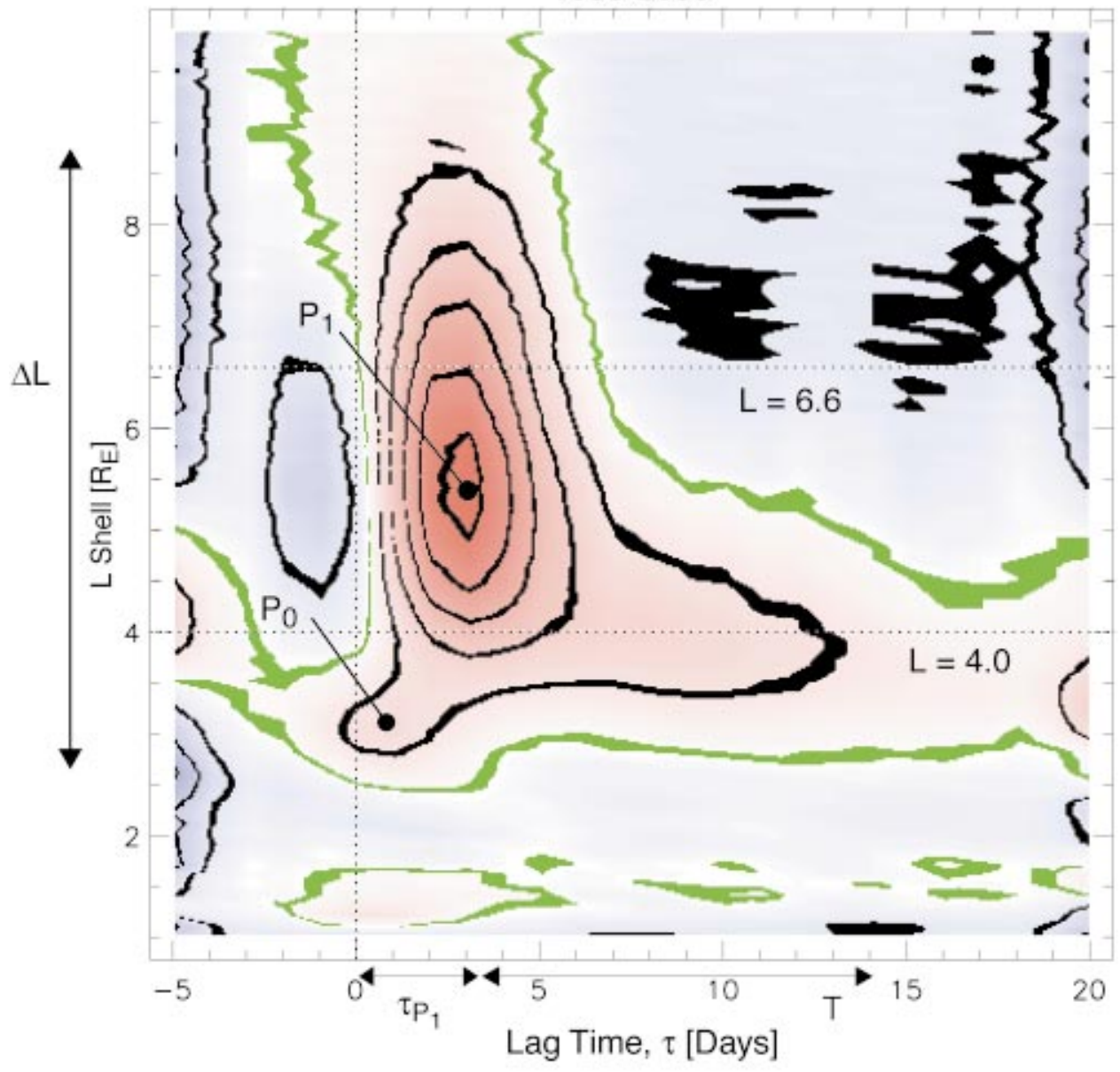

FIG. 3. (Color) Impulse response function parametrized by $L$ shell shown as a function of both lag time and $L$. Two of the regions, $P_{0}$ and $P_{1}$ are pointed out. Note the difference in response time. Horizontal dotted lines mark the $L$ shells 4.0 ("heart" of the outer zone) and 6.6 (geosynchronous orbit, where most of the historical observations have been made).

\section{$\mathrm{H}(\mathrm{t} ; \mathrm{L}) \times 100\left[\# / \mathrm{cm}^{3} / \mathrm{sr} / \mathrm{sec} 1 /\right.$ day $]$

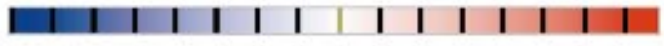

$\begin{array}{lllll}-0.20 & -0.10 & 0.00 & 0.10 & 0.20\end{array}$

$P_{1}$. Indeed, both aforementioned observations were made during magnetic cloud passages. ${ }^{12,13}$

Thus peaks $P_{0}$ and $P_{1}$ constitute distinct global modes of coupling to the solar wind and energy transfer to the magnetosphere. They occur at very different spatial regions of the inner magnetosphere, and at clearly different times after solar wind impact. Although the filter approach does not indicate which are the physical processes responsible for this coupling, it determines the time scales and interaction regions.

There are probably several more global modes of response than $P_{0}$ and $P_{1}$. A distinct type of response, called $P_{2}$, is identified in regions $L>7 R_{E}$ as discussed below. More important, it is conceivable that, in the context of the inner magnetosphere constitutes a nonlinear plasma system, under certain conditions these modes may be excited individually. That means that there may be preferential excitation of each mode by a suitable solar wind driver. We examine that possibility next. ${ }^{23}$

\section{SUPERPOSED EPOCH-ANALYSIS: SOLAR WIND PRECURSORS}

A significant part of understanding how the relativistic electron population in the inner magnetosphere is energized, is related to the nature of the most geoeffective solar wind conditions that drive such an energization. Given the comprehensive coverage by SAMPEX it is also possible to examine what are geoeffective conditions for each $L$ shell separately. We will address these two questions using a superposed-epoch analysis of solar wind intervals. This superposed epoch analysis is similar to the approach used by many researchers earlier. Perhaps the most interesting results are those by O'Brien et al. ${ }^{24}$ who obtained conditions in the solar wind for prolonged vs short-duration conditions of electron storms.

Here, events in the solar wind will be selected based on a function of the flux $j(t ; L)$ resulting from the geoeffective 

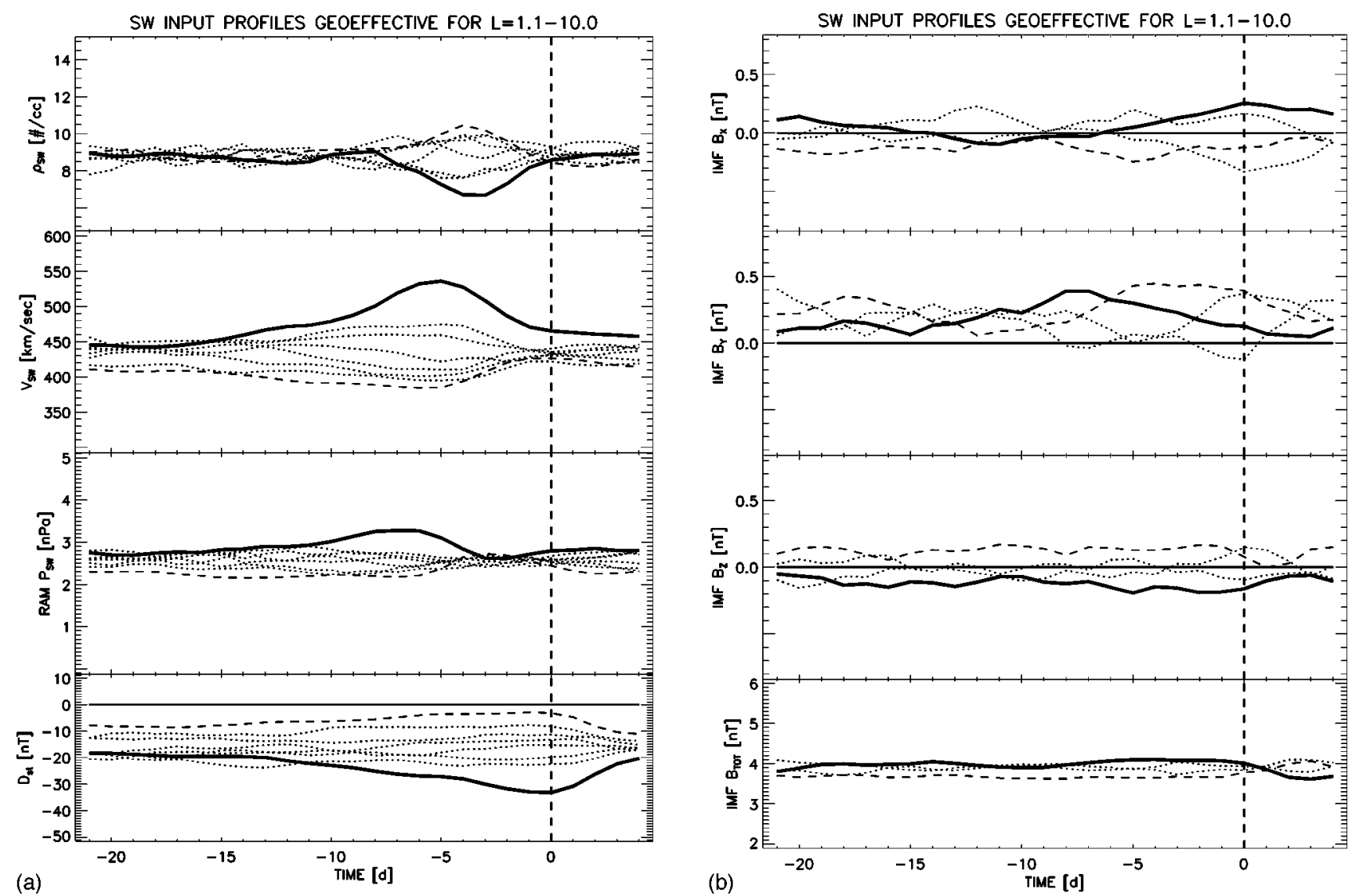

FIG. 4. Superposed-epoch activity: profiles of the globally-geoeffective precursor. Here "globally" means in the entire inner magnetosphere. The epoch $(\tau=0)$ is defined by the time index of $j_{\mathrm{RMS}}(t)$ [see Eq. (7)]. (a) Solar wind number density (measured in particles per cc), velocity, and ram pressure, as well as Dst. Each graph shows solar wind profiles for 8 levels of geoeffectiveness. Geoeffectiveness is determined as the global activity index (7) evaluated at $\tau=0$. Heavy lines: most geoeffective. Dashed: least geoeffective. The peak of the heavy line for $V_{\mathrm{SW}}$ at $\tau=-5$ days indicates that the most geoeffective structure is a high-speed stream (see also text). (b) Interplanetary magnetic field (IMF) components and field magnitude. Each graph shows IMF profiles for 4 levels of geoeffectiveness. The most goeffective $B_{z}$ profile is negative because it induces magnetic reconnection and a first stage of particle acceleration through various regimes of convection.

solar wind. If Eq. (3) is discretized in time, it can be rewritten as an inner product,

$$
j(t ; L)=\mathbf{H} \cdot \mathbf{I}^{\mathrm{TR}}(t),
$$

where $C$ and $\varepsilon(t)$ have been omitted, and vectors $\mathbf{H}$ and $\mathbf{I}$ are

$$
\begin{aligned}
\mathbf{H}= & {\left[h_{T_{s}}, h_{T_{s+1}-1}, \ldots, h_{0}, h_{1}, \ldots, h_{T}\right], } \\
\mathbf{I}(t) \equiv & {\left[V_{\mathrm{SW}}(t-T), V_{\mathrm{SW}}(t-T+1), \ldots, V_{\mathrm{SW}}(t-1),\right.} \\
& \left.V_{\mathrm{SW}}(t), \ldots, V_{\mathrm{SW}}\left(t+T_{s}\right)\right] .
\end{aligned}
$$

The superscript TR indicates time reversal of $\mathbf{I}$.

The vector $\mathbf{I}(t)$ is the solar wind precursor of an electron storm. The precursor has a certain geoeffectiveness, i.e., it produces an electron flux $j(t ; L)$ on day $t$. We distinguish between global and local (restricted in $L$ ) measures of activity.

\section{A. Global activity}

We define an index of global activity in order to classify the solar wind precursors,
Daily Global RMS Flux: $j_{\text {RMS }}(t)$

$$
=\left(\frac{1}{L_{\max }-L_{\min }} \int_{L_{\min }}^{L_{\max }} j_{e}^{2}(t ; L) d L\right)^{1 / 2},
$$

where from the SAMPEX orbit we have $L_{\min }=1, L_{\max }$ $=10 R_{E}$ (see also Refs. 1, 21).

We classify the activity over the 8 years into $k=4$ activity levels (most geoeffective, moderately, low, and not geoeffective) with equal numbers of $(j, \mathbf{I})$ data. For smoothlyvarying variables we use $k=8$ or 16 levels. The precursors $\mathbf{I}(t)$ for each activity level are superposed and averaged (superposed-epoch analysis). The epoch (relative time) is determined by the flux, $j(t)$. The same averaging is performed for the density, pressure, IMF $B_{z}$ component, IMF magnitude, and $D_{\text {st }}$ profiles.

The average profiles of the solar wind precursors are shown in Fig. 4. For each solar wind variable the most geoeffective profile is denoted by a heavy solid line. The least geoeffective profile is denoted by a dashed line. In Fig. 4(a), the velocity profile has a baseline amplitude of $435 \mathrm{~km} / \mathrm{s}$ and increases to $536 \mathrm{~km} / \mathrm{s} 5$ days before impact time, $\tau=0$. The 

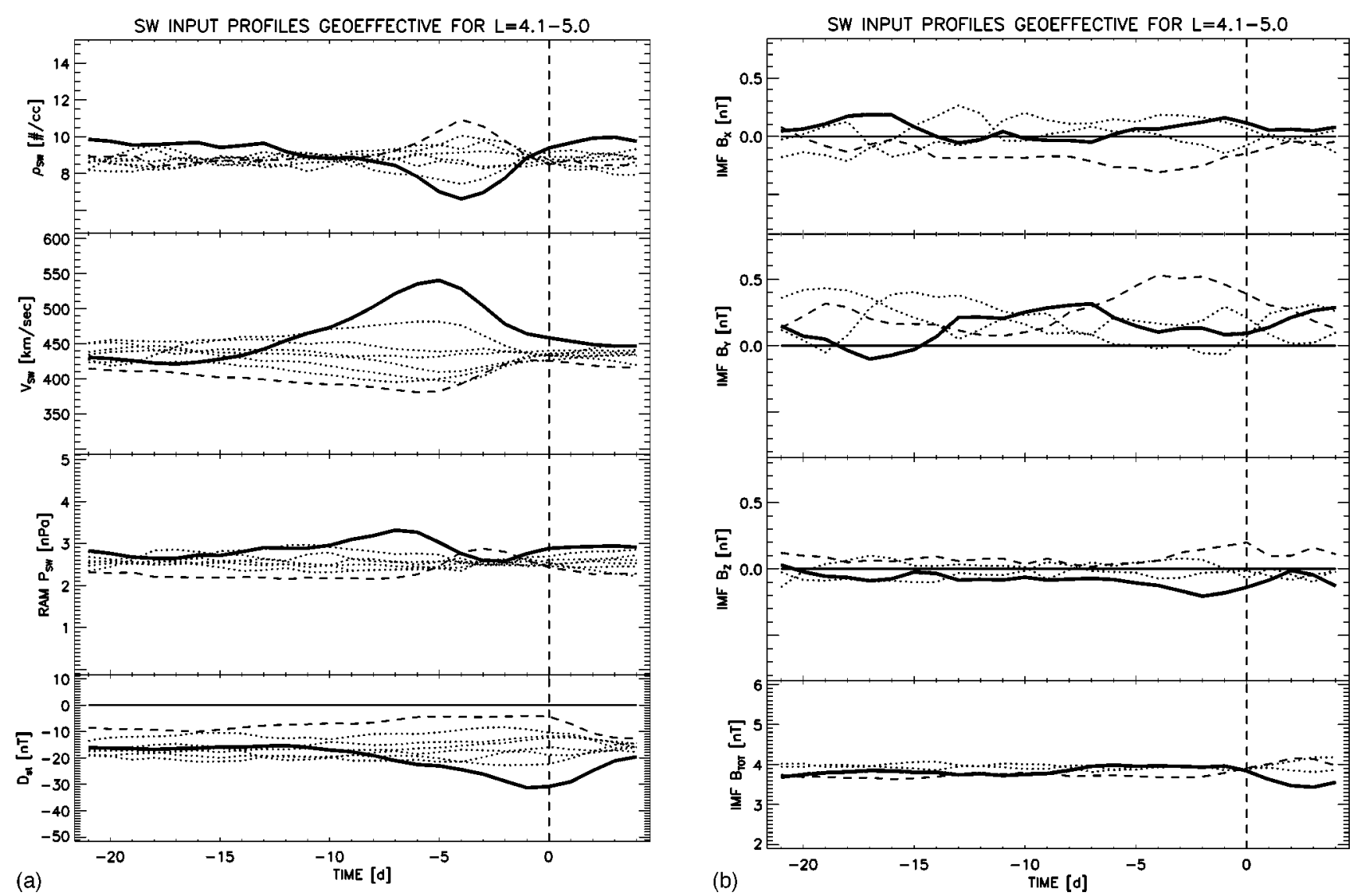

FIG. 5. Superposed-epoch activity: locally geoeffective precursors for $L=4.1-5.0$. Profiles are denoted for their geoeffectiveness [the local activity index (9) at $\tau=0$ ] as in Fig. 4. The strong similarity with the profiles geoeffective for $j_{\mathrm{RMS}}(t)$ (Fig. 4) is because fluxes at $L<4<5$ are much higher than in any other $L$ shell, and therefore dominate the averaging of Eq. (7). This means that here, too, the most geoeffective structures are high-speed streams.

temporal duration indicates that this profile is an average of mostly solar wind streams. The amplitude is lower than typical streams because it is averaged over a very large number of observations ( $k=8$ so $N=2901 / k=363$ ) and the distribution is similar to a power-law or a log-normal. If we choose a higher number of levels (e.g., $k=32$ ) the profiles are averaged over fewer instances, so their amplitude increases to levels more representative of high-speed streams.

As the $V_{\text {Sw }}$ profile increases several days before impact, the plasma density decreases. The plasma pressure for the intervals of most geoeffective inputs is $2.872 \mathrm{nPa}$, up $12 \%$ from its long-term average (2.573 nPa) as Fig. 4(a) shows. About 10 days before the electron acceleration the pressure starts increasing, reaching eventually $14 \%$ higher than average, and 5 days before the acceleration it starts dropping, reaching a minimum $9 \%$ below the average 2 days before the electron storm. The amplitude of the pressure pulse grows if we divide the data into greater numbers of levels $(k)$.

In terms of magnetic field profiles, the IMF $B_{z}$ component is negative for the most geoeffective profile, and positive for the least geoeffective [Fig. 4(b)]. Here we use $k=4$ levels because of the higher variability of the field compared to the solar wind plasma parameters. On the other hand, the field magnitude $|B|$ is somewhat elevated (4\%) from its longterm average value of $4.028 \mathrm{nT}$. The magnitude increases steadily for $>15$ days prior to the electron event by $25 \%$ from its earliest value. Then it decreases to its original levels within 4 days.

In summary here are the properties of the globally most geoeffective IMF/solar wind prior to impact:
Most Globally-Geoeffective precursor (stream-like) $-5<\tau<-2$ days

$$
\begin{gathered}
\rho_{\mathrm{SW}}^{(\min )}(\tau)=6.67 \mathrm{No} . / \mathrm{cm}^{3}, \\
V_{\mathrm{SW}}^{(\max )}(\tau)=536 \mathrm{~km} / \mathrm{s}, \\
\left\{\begin{array}{l}
P_{\mathrm{SW}}^{(\max }(\tau)=3.279 \mathrm{nPa}, \\
P_{\mathrm{SW}}^{(\min )}(\tau)=2.611 \mathrm{nPa},
\end{array}\right. \\
B_{z}^{(\min )}(\tau)=-0.242 \mathrm{nT}, \\
|B(\tau)|^{(\min )}=4.36 \mathrm{nT} .
\end{gathered}
$$



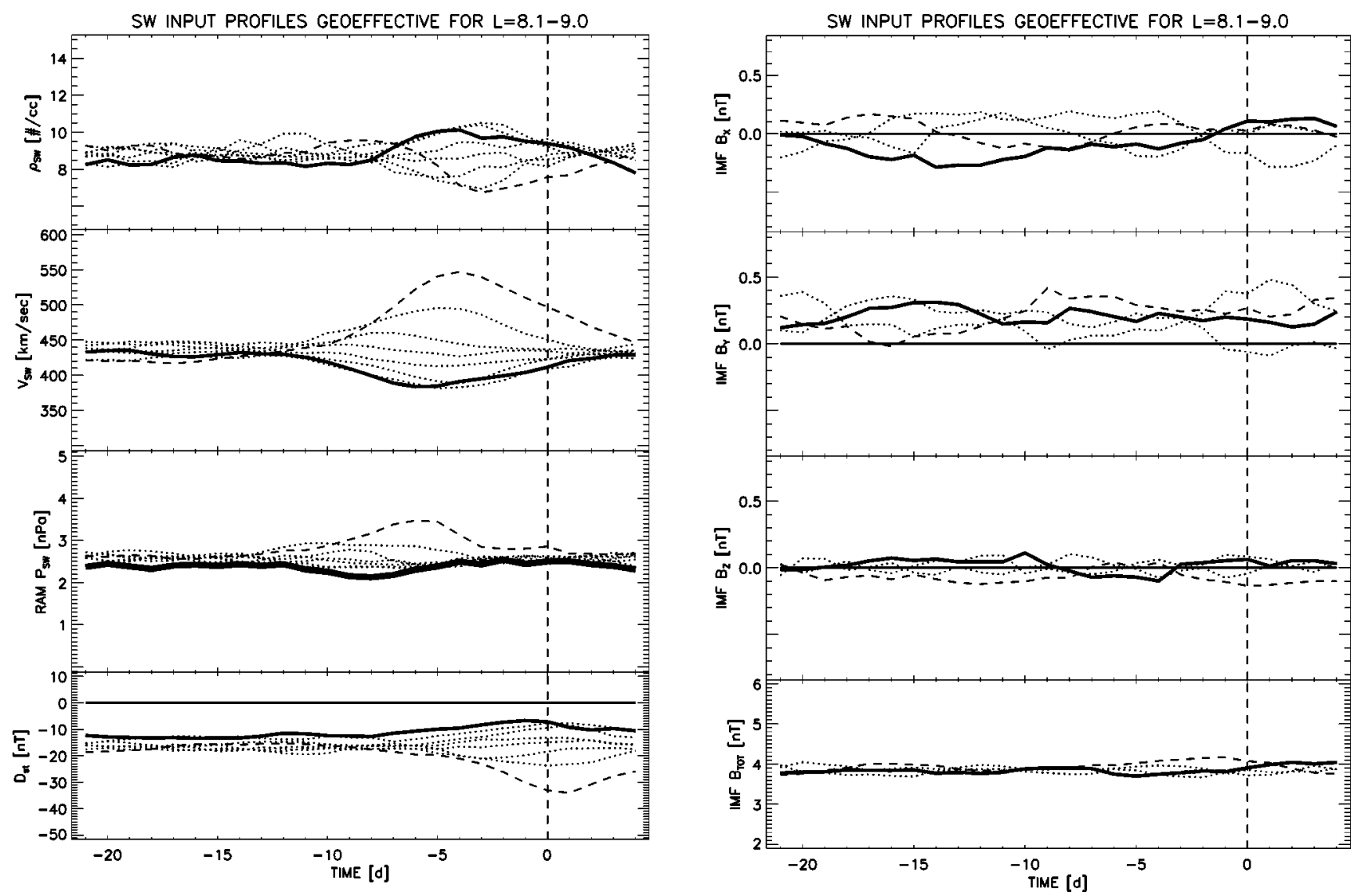

FIG. 6. As in Fig. 5, but for $L=8.1-9.0$. Note that the most geoeffective precursors (heavy lines) are convex exactly whyere the precursors of Figs. 4 and 5 are concave, and vice versa. The same holds for the least-geoeffective precursors. In particular, the most geoeffective IMF $B_{z}$ is positive, which may imply that instead of reconnection occurring on the dayside and close to the equator, it occurs on the nightside at high altitudes, poleward of the magnetic cusp.

These are to be compared with the average conditions in Eq. (2).

Note on statistical confidence levels: It should be noted that the standard deviations of the superposed-epoch analyses are large compared to the averages. Also generally the distributions of interplanetary parameters are not Gaussian. However, the number of samples in each distribution that are available for averaging is relatively high (typically $\sim 360$ or $\sim 720$ depending on $k$ ), and the distributions are reasonably close to well-defined distributions such as, e.g., log normal or power-law (see also remarks in Ref. 1). The confidence level in the superposed epoch analysis is high because of (a) the stationarity of the averages in time. Dividing the data in several years and repeating the analysis gives similar results. (b) Also, as will be seen in the next section, the solar wind precursors differ little within each region. Since the resolution in $L$ shell is high $\left(\Delta L=0.1 R_{E}\right)$, there are many independent measurements in each of the three regions that corroborate results for different radial distances.

\section{B. Locally geoeffective precursors}

We now focus our attention to the effects of the solar wind in a narrow radial range rather than the entire inner magnetosphere. Similar to the above approach we construct a daily flux index in a restricted range of $L$ shells,
Daily RMS Flux at $L_{1}: j_{\mathrm{RMS}}^{\left(L_{1}\right)}(t)$

$$
=\left(\frac{1}{\Delta L} \int_{L_{1}}^{L_{1}+\Delta L} j_{e}^{2}(t ; L) d L\right)^{1 / 2} .
$$

The activity levels are again defined by dividing the probability distribution of $j_{\mathrm{RMS}}^{(L)}$ in bins with equal numbers of data (again generally $k=4$ ).

In this case we find that the solar wind precursors for flux at radial distances from $L=3$ to $L=10 R_{E}$ are divided in three regions, $P_{0}, P_{1}$, and $P_{2}$. Precursors for the first two regions are qualitative similar to each other. Precursors for $P_{1}$, in particular, are virtually identical to the globally geoeffective precursors (8). However, the waveforms of precursors for $P_{2}$ are convex exactly where the precursors of $P_{0}$ and $P_{1}$ are concave, and vice versa. This holds both for the most-geoeffective and the least-geoeffective precursors.

\section{1. $P_{1}$ region}

The precursors for $P_{1}$ are shown in Fig. 5. It can be seen that the precursors for $P_{1}$ are virtually identical to the precursors of global activity [Fig. 4 and Eq. (8)], except for small quantitative variations. The most geoeffective precursors are characterized by increases in solar wind speed at $\tau$ $<-2$, simultaneous decreases of the density so that the pressure is approximately constant. The $B_{z}$ component is nega- 

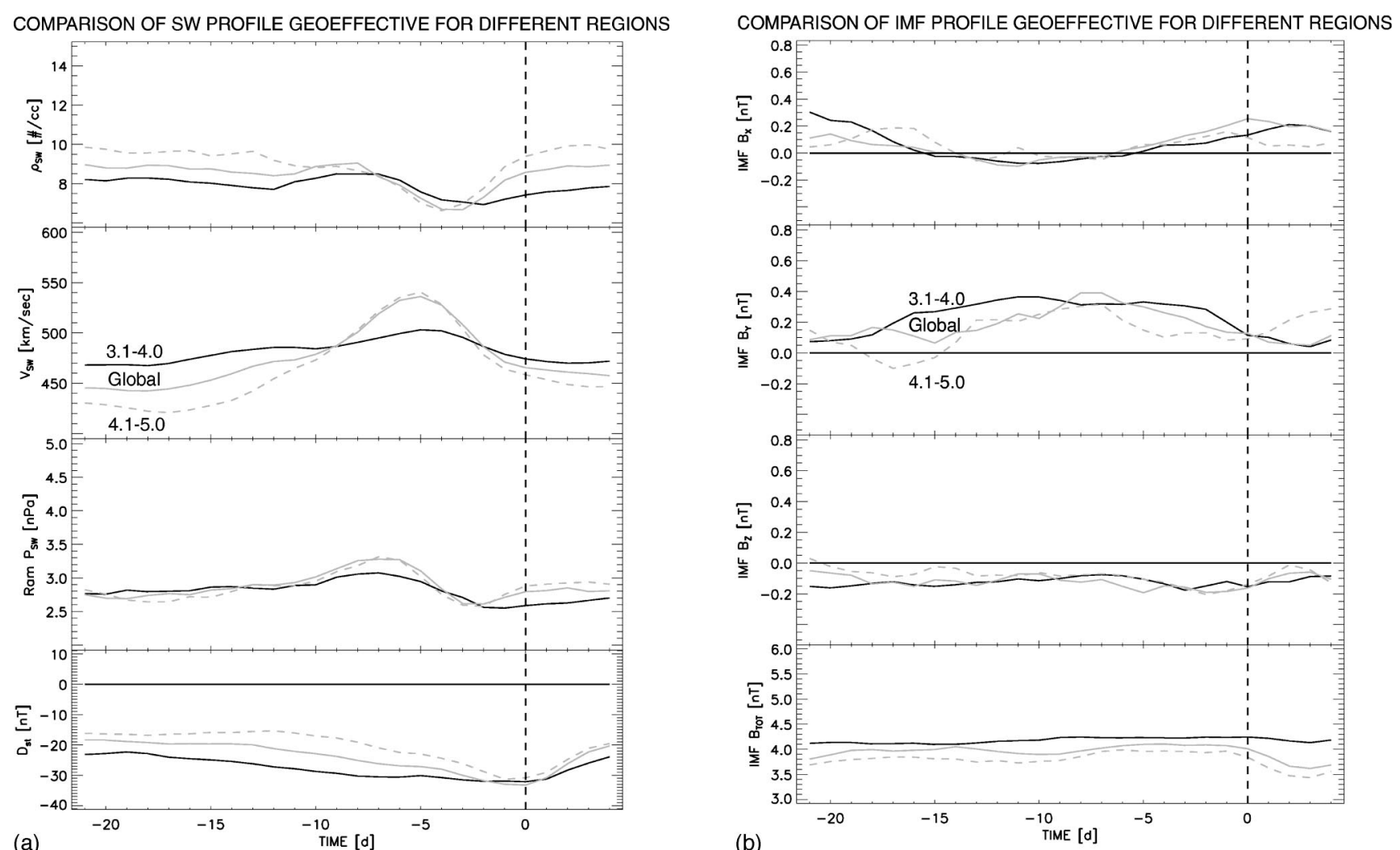

FIG. 7. Comparison between most-geoeffective precursor in $L=3.1-4.0$, the globally geoeffective precursor, and that for region $L=4.1-5.0$ (the last two are shown in Figs. 4 and 5, respectively). Superposed-epoch averages for solar wind plasma, $D_{\text {st }}$, and IMF variables are shown as in Fig. 4.

tive and the magnitude increases. The great similarity to Eq. (8) is not suprising, however, region $P_{1}$ contains by far most of the trapped particle content of the outer zone of the radiation belt and therefore dominates the global properties (region $P_{0}$ contains most of the remaining trapping capacity).

\section{2. $P_{2}$ region}

The precursors for this region are opposite from those of $P_{1}$. Increases in velocity are least geoeffective, while decreases are associated with transient enhancements of flux (Fig. 6). It seems that this region responds more to pressure (or density) increases.

It is perhaps more suprising that the most geoeffective magnetic field component is positive rather than negative [Fig. 6(b)]. Thus the most probable magnetic reconnection is probably less involved in accelerating particles in this region. This "most probable" magnetic reconnection is initiated by the IMF $B_{z}$ component turning negative, or antiparallel to the terrestrial field at the equatorial plane; the reconnection then takes place at low latitudes on the dayside of the magnetosphere. On the other hand, for positive $B_{z}$ reconnection can take place at high latitudes antisunward of the magnetic cusp. Due to the geometry, the positive- $B_{z}$ reconnection isless efficient in terms of geomagnetic effects. However, it may reveal or even enhance the role of the cusp in accelerating electrons. This may not be as unreasonable as it may sound: the role of the cusp in accelerating particles is well established. ${ }^{25}$ In fact recently it has been proposed that the fluxes generated by acceleration in the cusp may constitute a very significant source of radiation belt particles. ${ }^{26}$ In any case, the presence of a low-flux population at radial distances $L>7 R_{E}$, shown in Fig. 5 [and in fact as high as $L=20 R_{E}$ (Ref. 23)] is related to the plasma sheet on the nightside, and possibly to the cusp on the dayside.

The following table summarizes the most geoeffective inputs for this region:

Most Geoeffective Precursor for $P_{2}: \quad\left\{P_{\mathrm{SW}}^{(\max )}(\tau)=2.541 \mathrm{nP}\right.$,

$(7<L,-5<\tau<-2) \quad\left\{\quad\left\{P_{\mathrm{SW}}^{(\min )}(\tau)=2.131 \mathrm{nP}\right.\right.$,

$B_{z}^{(\max )}(\tau)=0.20 \mathrm{nT}$,

$|B(\tau)|^{(\mathrm{min})}=3.79 \mathrm{nT}$. 


\section{3. $P_{0}$ region}

The precursors for the innermost region do not appear to be qualitatively different from those of $P_{1}$ at the present time. However, there are small systematic differences. A comparison between the most geoeffective precursors of $P_{0}$, $P_{1}$, and the global precursors (8) are shown in Fig. 7. With regard to the solar wind plasma parameters [Fig. 7(a)] the $P_{0}$ region is less sensitive to increases in the solar wind velocity as its profile remains flat compared to the globally geoeffective response. Similarly the density and pressure are less pronounced than either the global response or the one geoeffective for $L=4.1-5.0 R_{E}$. Instead, the IMF seems to be a more significant input in this region [Fig. 7(b)], similarly to region $P_{2}$. The absolute magnitude of the field, $|B|$, is larger for $P_{0}$ than for either $P_{1}$ or the entire region. The difference between these amplitudes appears small, but in fact represents average values from a large number of events: approximately 360 for the solar wind plasma and Dst superposedepoch averages, and approximately 720 for the IMF superposed-epoch averages. As before, we use a large number for the magnetic field because its standard deviation is considerably higher than that of the plasma parameters.

\section{SUMMARY AND INTERPRETATION}

Collective effects dominate the dynamics of the inner magnetosphere plasma. ${ }^{27}$ They produce a coherent response of the inner magnetosphere to the solar wind input. ${ }^{28,29}$ The global coherence of the terrestrial magnetosphere is due to the relatively strong magnetic field which connects remote regions at fairly high fast-mode speeds. ${ }^{30}$

We have found that the response of the outer zone consists of different modes, of which we have identified three ( $P_{0}, P_{1}$, and $P_{2}$ ) here. Mode $P_{1}$ is characteristic for the region at approximately $4<L<7 R_{E}$ with a characteristic time scale of 2-3 days. On the other hand, $P_{0}$ takes place much earlier, at $t<1$, and at lower $L$ shells, $3<L<4 R_{E}$. The distinction of mode $P_{2}$ from the prototypical $P_{1}$ is evident in terms of their respective precursors (Fig. 5). The reason it is not seen as a separate mode in the impulse response $H(t ; L)$ (Fig. 3) is that we use the logarithmic flux $j$ (which decays relatively slowly with $L$ ); indeed if we use the original flux $J(t ; L)$ the response above $L=7 R_{E}$ is readily distinguished as different from the responses below $L$ $=7 R_{E}$ (see Fig. 2 of Ref. 1). In a parallel paper we show that the $P_{2}$ region may extend to at least $L=20 R_{E}$ and its low flux and other dynamics relate it most probably to the plasma sheet and possibly to the cusp. ${ }^{23}$ From that and other considerations it becomes evident that the sharp separation at $L \cong 7 R_{E}$ is related to the effect of the dayside magnetospheric boundary, typically at $L=8-10 R_{E}$ (and dynamically depending on the interplanetary plasma pressure).

Each one of the three modes responds to a different combination of solar wind precursors. Clearly all locations in the inner magnetosphere respond to all solar wind inputs, but the presence of modes indicates that there are preferred (i.e., most geoeffective) drivers for each region. We have qualitatively described the precursors for $P_{1}$ and $P_{2}$ in Eqs. (8) and (10), respectively, and more quantitatively in Figs. 4, 5, and
6. In terms of prediction, identification of precursors is important as work has shown for geomagnetic storms caused by passing ICMEs. $^{31}$

These findings raise the question, how can a low- $\beta$ kinetic-dominated plasma exhibit this type of coherence and large-scale structure? Clearly there are many significant particle-acceleration processes operating simultaneously and often independently. In several locations, however, and under specific external drivers they cooperate to produce a coherent response. This response maximizes transport of the solar wind energy through the inner magnetosphere until its eventual transmission or dissipation.

\section{ACKNOWLEDGMENTS}

We thank D. N. Baker, M.-C. Fok, S. F. Fung, X. Li, and E. Rigler for several useful discussions. We also thank the staff of NSSDC for maintaining the OMNI database.

This research was funded by NASA/LWS and NSF/SW grants.

${ }^{1}$ D. Vassiliadis, A. J. Klimas, S. G. Kanekal, D. N. Baker, and R. S. Weigel, J. Geophys. Res. 107, 1383 (2002).

${ }^{2}$ X. Li and M. A. Temerin, Space Sci. Rev. 95, 569 (2001).

${ }^{3}$ X. Li, D. N. Baker, M. A. Temerin, D. Larson, R. P. Lin, E. G. D. Reeves, M. D. Looper, S. G. Kanekal, and R. A. Mewaldt, Geophys. Res. Lett. 24, 923 (1997).

${ }^{4}$ R. H. W. Friedel, G. D. Reeves, and T. Obara, J. Atmos. Sol.-Terr. Phys. 64, 265 (2002).

${ }^{5}$ G. Rostoker, S. Skone, and D. N. Baker, Geophys. Res. Lett. 25, 3701 (1998).

${ }^{6}$ M. K. Hudson, S. R. Elkington, J. G. Lyon, C. C. Goodrich, and T. J. Rosenberg, "Simulation of radiation belt dynamics driven by solar wind variations," in Sun-Earth Plasma Connections, edited by J. L. Burch, R. L. Carovillano, and S. K. Antiochos, Geophysical Monograph 109 (AGU, Washington, D.C., 1999).

${ }^{7}$ S. R. Elkington, M. K. Hudson, and A. A. Chan, "Resonant acceleration and diffusion of outer zone electron in an asymmetric geomagnetic field," J. Geophys. Res. (submitted).

${ }^{8}$ M. Engebretson, K.-H. Glassmeier, M. Stellmacher, W. J. Hughes, and H. Luehr, J. Geophys. Res. 103, 26271 (1998).

${ }^{9}$ G. A. Paulikas and J. B. Blake, "Effects of the solar wind on magnetospheric dynamics: energetic electrons at the sunchronous orbit," in Quantitative Modeling of Magnetospheric Processes, edited by W. P. Olson, Geophysical Monograph 21 (AGU, Washington, D.C., 1979).

${ }^{10}$ C. J. Farrugia, F. T. Gratton, and R. B. Torbert, Space Sci. Rev. 95, 443 (2001).

${ }^{11}$ V. A. Osherovich and L. F. Burlaga, "Magnetic clouds," in Coronal Mass Ejections, edited by N. Crooker, J. A. Joselyn, and J. Feynman, Geophysical Monograph 99 (AGU, Washington, D.C., 1997).

${ }^{12}$ D. N. Baker, T. I. Pulkkinen, X. Li, S. G. Kanekal, K. W. Ogilvie, R. P. Lepping, J. B. Blake, L. B. Callis, G. Rostoker, H. J. Singer, and G. D. Reeves, Geophys. Res. Lett. 25, 2975 (1998).

${ }^{13}$ G. D. Reeves, R. H. W. Friedel, R. D. Belian, M. M. Meier, M. G. Henderson, T. Onsager, H. J. Singer, D. N. Baker, X. Li, and J. B. Blake, J. Geophys. Res. 103, 17559 (1998).

${ }^{14}$ X. Li, I. Roth, M. Temerin, J. R. Wygant, M. K. Hudson, and J. B. Blake, Geophys. Res. Lett. 20, 2423 (1993).

${ }^{15}$ W. R. Cook, A. C. Cummings, J. R. Cummings, T. L. Garrard, B. Kecman, R. A. Mewaldt, R. S. Selesnick, E. C. Stone, D. N. Baker, T. T. Von Rosenvinge, J. B. Blake, and L. B. Callis, IEEE Trans. Geosci. Remote Sens. 31, 565 (1993).

${ }^{16}$ D. N. Baker, G. M. Mason, O. Figueroa, G. Colon, J. G. Watzin, and R. M. Aleman, IEEE Trans. Geosci. Remote Sens. 31, 531 (1993).

${ }^{17}$ W. H. Press, B. P. Flannery, S. A. Teukolsky, and W. V. Vetterling, $\mathrm{Nu}-$ merical Recipes: The Art of Scientific Computing, 2nd ed. (Cambridge University Press, Cambridge, 1993). 
${ }^{18}$ T. Nagai, Geophys. Res. Lett. 15, 425 (1988).

${ }^{19}$ D. N. Baker, R. L. McPherron, T. E. Cayton, and R. W. Klebesadel, J. Geophys. Res. 95, 15133 (1990).

${ }^{20}$ A. Tsutai, C. Mitsui, and T. Nagai, Earth, Planet. Space 51, 219 (1999).

${ }^{21}$ D. Vassiliadis, R. S. Weigel, S. G. Kanekal, A. J. Klimas, D. N. Baker, E. J. Rigler, and R. Mewaldt, "Response of the magnetospheric electron flux to the solar wind as a function of activity level and associated geoeffective interplanetary structures," J. Geophys. Res. (submitted).

${ }^{22}$ H. C. Koons and D. J. Gorney, J. Geophys. Res. 96, 5549 (1991).

${ }^{23}$ D. Vassiliadis, R. S. Weigel, A. J. Klimas, S. F. Fung, S. G. Kanekal, R. A. Mewaldt, D. N. Baker, and E. J. Rigler, "Structure of the electron radiation belt's outer zone," Geophys. Res. Lett. (submitted).

${ }^{24}$ T. P. O'Brien, R. L. McPherron, G. D. Reeves, R. Friedel, and H. J. Singer, J. Geophys. Res. 106, 15533 (2001).
${ }^{25}$ R. B. Sheldon, H. E. Spencé, J. D. Sullivan, T. A. Fritz, and J. Chen, Geophys. Res. Lett. 25, 1825 (1998).

${ }^{26}$ T. A. Fritz, J. S. Chen, and R. B. Sheldon, Adv. Space Res. 25, 1445 (2000).

${ }^{27}$ D. N. Baker, S. G. Kanekal, A. J. Klimas, D. Vassiliadis, and T. I. Pulkkinen, Phys. Plasmas 6, 4195 (1999).

${ }^{28}$ S. G. Kanekal, D. N. Baker, J. B. Blake, B. Klecker, R. A. Mewaldt, and G. M. Mason, J. Geophys. Res. 104, 24885 (1999).

${ }^{29}$ S. G. Kanekal, D. N. Baker, and J. B. Blake, J. Geophys. Res. 106, 29721 (2001).

${ }^{30}$ D. Vassiliadis, A. J. Klimas, J. A. Valdivia, and D. N. Baker, Adv. Space Res. 26, 197 (2000).

${ }^{31}$ J. Chen, P. J. Cargill, and P. J. Palmadesso, Geophys. Res. Lett. 23, 625 (1996). 